\title{
Modal Expansionism
}

\section{Alexander Roberts ${ }^{1}$}

Received: 16 October 2017 / Accepted: 30 March 2019 / Published online: 1 June 2019

(c) The Author(s) 2019

\begin{abstract}
There are various well-known paradoxes of modal recombination. This paper offers a solution to a variety of such paradoxes in the form of a new conception of metaphysical modality. On the proposed conception, metaphysical modality exhibits a type of indefinite extensibility. Indeed, for any objective modality there will always be some further, broader objective modality; in other terms, modal space will always be open to expansion.
\end{abstract}

Keywords Metaphysical necessity $\cdot$ Indefinite extensibility $\cdot$ Bimodal logic

Call a modality objective when it is non-epistemic, non-deontic, non-linguistic, and non-intentional. The objective modalities are roughly the modalities expressed by circumstantial modal terms. ${ }^{1}$ We routinely use such terms in natural language to express various practical modalities, and, on occasion, nomological modality. Yet, although uncommon in natural language, circumstantial modal auxiliary verbs like 'must' can be used unrestrictedly, that is without restriction to any set of circumstances; on this reading, 'it must be that $\phi$ ' is true when $\phi$ is the case whatever the circumstances. When read as such, the objective modality expressed by the modal is metaphysical modality. Metaphysical modality is accordingly the maximal objective modality: possibility according to any objective modality entails possibility according to metaphysical modality. ${ }^{2}$

\footnotetext{
${ }^{1}$ The term 'objective modality' was introduced in the relevant sense by Williamson [28], who connects it to Angelika Kratzer's [12, chap. 2] notion of a circumstantial modal expression. A rough test for whether a modal term is circumstantial is whether it is sensitive to the mode under which a proposition in its scope is presented.

${ }^{2}$ Some philosophers might be tempted by the idea that metaphysical modality is in some sense a restriction of logical modality. This is compatible with the claim that metaphysical modality is the maximal objective modality, for logical modality is a linguistic modality and thus not objective in the relevant sense.
}

Alexander Roberts

alexander.roberts@philosophy.ox.ac.uk

1 Faculty of Philosophy, University of Oxford, Oxford, UK 
The idea that metaphysical modality is the maximal objective modality is widespread. It is frequently assumed in philosophical discussion and regularly appealed to in explanations of the nature of metaphysical necessity. It reflects the popular thought that metaphysical modality in some sense concerns all possible worlds. Nevertheless, this paper argues for a new conception of metaphysical modality according to which there is one sense in which this maximality thesis is false. According to the proposed conception, for any objective modality there will always be some further, broader objective modality; or, in other terms, metaphysical modal space will always be open to expansion.

The structure of the paper is as follows. Sections 1 and 2 present a modal recombinatorial paradox due to Kit Fine [4, pp. 223-224], later reformulated by Peter Fritz [8]. ${ }^{3}$ Section 3 outlines the parallels which this recombinatorial paradox bears to the paradoxes of set theory, and suggests a treatment of the former similar to extant treatments of the latter. Using the resources of a novel bimodal logic, Section 4 develops the suggested treatment and its revisionary modal metaphysic. Sections 5 and 6 respectively discuss objections to and further applications of the newly developed metaphysics of modality.

\section{Recombination and Paradox}

Consider the property of being an elementary particle. This property is intrinsic and therefore it can be possessed independently of whether it is possessed by any other individuals. As a result, the following modal recombinatorial principle will be true (where the operative modality is metaphysical modality):

(R1) For any possible world $w$, there is a possible world $u$ where all elementary particles at $w$ are elementary particles and there is an elementary particle distinct from every elementary particle at $w$.

(R1) is plausible because in denying it one objectionably places arbitrary limits on the extent of modal space. Given the purported utterly liberal character of metaphysical modality, for every world $w$ there should be some possible world $u$ where all elementary particles at $w$ are all elementary particles, and there is some elementary particle distinct from every elementary particle at $w$. After all, could not all elementary particles at $w$ not be elementary particles with some distinct elementary particle? If $w$ contains no elementary particles, surely it is metaphysically possible that there be elementary particles. If $w$ contains one or more elementary particles, surely it is metaphysically possible that there be all of them plus a duplicate of one of them.

Now consider another plausible recombinatorial principle:

(R2) There is some possible world $w$ such that for all possible worlds $u$ all elementary particles at $u$ are elementary particles at $w$.

\footnotetext{
${ }^{3}$ Strictly speaking, Fine and Fritz's paradoxes are closely related but not one and the same; Fine's puzzle involves classes of possibilia, whereas Fritz's puzzle involves no such resources.
} 
(R2) articulates the idea that there is a possibility in which all elementary particles across modal space are elementary particles. It too is motivated by an aversion to imposing arbitrariness onto modal space. Since something's being an elementary particle is compossible with some other thing's also being an elementary particle, there appears to be no limit as to which possible elementary particles are compossible. Again, given the purported utterly liberal character of metaphysical modality, it should not be the case that, for example, it is possible that all elementary particles across modal space except one are elementary particles, yet not possible that all elementary particles across modal space are elementary particles. To admit that type of division would be to admit a type of objectionable arbitrariness which a theory of metaphysical modality theory ought to eschew. ${ }^{4}$

However, despite (R1) and (R2) both being plausible modal recombinatorial principles, Kit Fine [4, pp. 223-224] presents an argument that similar such principles together lead to paradox. To adapt Fine's argument, consider $w$ in (R2). From (R1) we can infer that:

(C) There is a possible world $u$ where all elementary particles at $w$ are elementary particles and there is also a elementary particle which is not identical with any of them.

But all the elementary particles at $w$ are all the elementary particles at all worlds. Contradiction. As a result, (R1) and (R2) cannot be satisfied by a single modality, for no one modality can possesses this extreme degree of recombinatorial liberality, including metaphysical modality.

\section{Reformulation}

The above formulation of the paradox employed first-order quantification over possible worlds and possibilia. But one should not assume that the paradox is generated by an extensional reduction of modal notions. Indeed, Peter Fritz [8] has recently shown how to formalise (R1), (R2), and (C) in the language of pure quantified modal logic, which avoids first-order quantification over worlds and possibilia. This should dispel any concern that the paradox is an artefact of reductionism about modal notions.

For a quantified modal language to have the expressive capacity required to formulate the principles, it must be capable of simulating first-order quantification over possible worlds and possibilia, and simulating cross-reference to worlds over which one has previously 'considered'. This expressive requirement is met by enriching the vocabulary of a conventional quantified modal language with infinitely many distinct pairs of indexed Vlach-operators $\left(\uparrow^{n}, \downarrow^{n}\right.$, for every natural number $n$ )-operators

\footnotetext{
${ }^{4}$ Following David Lewis's [13, pp. 101-104] discussion, advocates of concrete modal realism might reject (R2) on the basis that some, perhaps large infinite cardinal is an upper bound on the possible size of spacetime. (In his discussion, Lewis is aware that this would still involve some degree of arbitrariness; see Lewis (ibid., p.103) in particular.) But this response does not seem to address the core concern, for it is surely metaphysically possible that elementary particles can be colocated, just as some philosophers, such as Hawthorne and Uzquiano [10, p. 55], have claimed bosons can actually be.
} 
that allow one to 'store' a world at a place on a list, and at a later point 'retrieve' that world as the world of evaluation. Specifically, $\uparrow^{n}$ stores the current world of evaluation at the $n$th place on a list, and $\downarrow^{n}$ places the world stored at the $n$th place on the list as the world of evaluation. To give an example of how the operators work, consider the following formula:

$$
\diamond \uparrow^{1} \square \forall x\left(F(x) \rightarrow \downarrow^{1} G(x)\right)
$$

This can be read in extensional parlance as the claim that there is some possibility at which every $F$ in any possible world is a $G$.

To be more precise, the exact vocabulary of the quantified modal language $\left(=\mathcal{L}_{1}\right)$ in which we shall work consists of countably many individual variables $\left(x_{1}, x_{2}, \ldots\right)$, countably many predicate letters $(E, F, G \ldots), \neg$ (negation) and $\rightarrow$ (material conditional) as the only truth-functional connectives, $\forall$ (universal quantification) as the sole quantifier, and the modal operators $\square$ (necessity), $\uparrow^{n}$ and $\downarrow^{n}$ for every natural number $n$. The existential quantifier $\exists$ and possibility operator $\diamond$ are used as abbreviations for $\neg \forall \neg$ and $\neg \square \neg$, respectively.

Following Kit Fine's [3, pp. 135-136] early work, Fabrice Correia [1] has shown how to provide a model-theoretic semantics for languages like $\mathcal{L}_{1}$. We follow his lead. Thus a frame $\mathfrak{F}$ is an ordered pair $\langle\mathcal{W}, \mathcal{D}\rangle$, where $\mathcal{W}$ is a non-empty set understood as a non-empty set of worlds, and $\mathcal{D}$ is a function taking each $w \in \mathcal{W}$ into a nonempty set $\mathcal{D}_{w}$, understood as that world's domain. ${ }^{5}$ A model $\mathfrak{M}$ of $\mathcal{L}_{1}$ based on $\mathfrak{F}=$ $\langle\mathcal{W}, \mathcal{D}\rangle$ is a triple $\langle\mathcal{W}, \mathcal{D}, \mathcal{V}\rangle$, where $\mathcal{V}$ is a function taking any individual constant of the language into a member of $\cup_{w \in \mathcal{W}} \mathcal{D}_{w}$, and any $n$-place predicate letter of the language and world into a set of $n$-tuples of $\cup_{w \in \mathcal{W}} \mathcal{D}_{w}$, that predicate's extension at that world. Given a model, an assignment is a function $g$ mapping each individual variable of the language to a member of $\cup_{w \in \mathcal{W}} \mathcal{D}_{w}$. Where $x$ is an individual variable, two assignments $g$ and $g^{\prime}$ are $x$-alternatives iff $g$ and $g^{\prime}$ assign the same values for all variables, except possibly $x$.

For a set $S$, let $\mathcal{S}{ }^{<\omega}$ be the set of $\omega$-sequences of members of $\mathcal{S}$. For seq $\in \mathcal{S}^{<\omega}$ and $s \in \mathcal{S}, s e q^{n \rightarrow s}$ is the sequence obtained from replacing the $n$th item in $s e q$ with $s$, and $\operatorname{seq}(n)$ is the $n$th item in seq.

We define ' $(\mathfrak{M}$, seq, $w) \models_{g} A$ ' for $\mathfrak{M}=\langle\mathcal{W}, \mathcal{D}, \mathcal{V}\rangle$, seq $\in \mathcal{W}^{<\omega}, w \in \mathcal{W}, g$ an assignment to the variables in $\mathcal{M}$, and $A$ an formula of the language, recursively as follows $\left(\mathcal{V}_{g}(a)\right.$ is used for $g(a)$ if $a$ is an individual variable and for $\mathcal{V}(a)$ if $a$ is an individual constant):

$(\mathfrak{M}$, seq,$w) \models_{g} F a_{1} \ldots a_{n}$ iff $\left\langle\mathcal{V}_{g}\left(a_{1}\right), \ldots, \mathcal{V}_{g}\left(a_{n}\right)\right\rangle \in \mathcal{V}(F, w)$;

$(\mathfrak{M}$, seq,$w) \models_{g} \neg A$ iff $(\mathfrak{M}$, seq, $w) \not \models_{g} A$;

$(\mathfrak{M}$, seq,$w) \models_{g} A \rightarrow B$ iff either $(\mathfrak{M}$, seq,$w) \models_{g} A$ or $(\mathfrak{M}$, seq, $w) \models_{g} B$;

$(\mathfrak{M}$, seq, $w) \models_{g} \forall x A$ iff $(\mathfrak{M}$, seq, $w) \models_{g^{\prime}} A$ for every $x$-alternative $g^{\prime}$ of $g$ such that $g^{\prime}(x) \in \mathcal{D}_{w}$;

\footnotetext{
${ }^{5}$ Since frames contain no accessibility relations, the model theory validates an $\mathbf{S 5}$ logic for metaphysical necessity, i.e. $\square$. This is a harmless simplifying assumption, and could be dropped for current purposes. (For example, as Fritz [8, Appendix C] notes, the inconsistency remains even if the 4 axiom for metaphysical necessity is rejected.)
} 
$(\mathfrak{M}$, seq,$w) \models_{g} \square A$ iff for every $w^{\prime} \in \mathcal{W},\left(\mathfrak{M}\right.$, seq,$\left.w^{\prime}\right) \models_{g} A$;

$(\mathfrak{M}$, seq,$w) \models_{g} \uparrow^{n} A$ iff $\left(\mathfrak{M}, \operatorname{seq}^{n \rightarrow w}, w\right) \models_{g} A$;

$(\mathfrak{M}, \operatorname{seq}, w) \models_{g} \downarrow^{n} A$ iff $(\mathfrak{M}, \operatorname{seq}, \operatorname{seq}(n)) \models_{g} A$.

A formula $A$ is true at $\langle\operatorname{seq}, w\rangle$ for $\operatorname{seq} \in \mathcal{W}^{<\omega}$ and $w \in \mathcal{W}$ iff for all variable assignments $g$, $(\mathfrak{M}, \operatorname{seq}, w) \models_{g} A$. A formula $A$ of $\mathcal{L}_{1}$ is true in an $\mathcal{L}_{1}$ model $\mathfrak{M}=\langle\mathcal{W}, \mathcal{D}, \mathcal{V}\rangle$ iff it is true at $\langle$ seq, $w\rangle$, for every seq $\in \mathcal{W}^{<\omega}$, and every $w \in \mathcal{W}$. A formula $A$ of $\mathcal{L}_{1}$ is valid iff it is true in every $\mathcal{L}_{1}$ model. A set $\Gamma$ of $\mathcal{L}_{1}$ formulas entails an $\mathcal{L}_{1}$ formula $A$ iff for every $\mathcal{L}_{1}$ model $\mathfrak{M}=\langle\mathcal{W}, \mathcal{D}, \mathcal{V}\rangle$, every seq $\in \mathcal{W}^{<\omega}$, $w \in \mathcal{W}$, and every variable assignment $g$, if $(\mathfrak{M}$, seq, $w) \models_{g} \gamma$ for each $\gamma \in \Gamma$, then $(\mathfrak{M}$, seq,$w) \models_{g} A$. For convenience, we shall talk of some $\mathcal{L}_{1}$ formulas (as opposed to their set) entailing another $\mathcal{L}_{1}$ formula.

As Fritz [8] shows, we can use the above apparatus to formalise (R1), (R2), and (C) in the enriched modal language $\mathcal{L}_{1}$, thus avoiding first-order quantification over worlds. Letting ' $E$ ' denote the property of being an elementary particle, we write:

$$
\square \uparrow^{1} \diamond \uparrow^{2}\left(\downarrow^{1} \forall x\left(E(x) \rightarrow \downarrow^{2} E(x)\right) \wedge \exists x\left(E(x) \wedge \downarrow^{1} \neg E(x)\right)\right)
$$

$$
\diamond \uparrow^{1} \square \forall x\left(E(x) \rightarrow \downarrow^{1} E(x)\right)
$$

Yet (R1*) entails that $\square \uparrow^{1} \diamond \uparrow^{2} \exists x\left(E(x) \wedge \downarrow^{1} \neg E(x)\right)$-informally, this is because both conjuncts of a necessarily possibly true conjunction are necessarily possible. Since $\uparrow^{2}$ is redundant in this formula it simplifies as follows:

$$
\square \uparrow^{1} \diamond \exists x\left(E(x) \wedge \downarrow^{1} \neg E(x)\right)
$$

Yet this is not satisfiable with $(\mathrm{R} 2 *)$, in the sense that it cannot be true in any $\mathcal{L}_{1}$ model in which (R2*) is.

Accordingly, the paradox can be formulated in an appropriately expressive quantified modal language. Thus $(\mathrm{R} 1) /(\mathrm{R} 1 *)$ and $(\mathrm{R} 2) /(\mathrm{R} 2 *)$ cannot both be licensed by a single notion of metaphysical necessity. But the grounds for accepting either principle are essentially the same: both principles are motivated by the fact that rejecting them would be to admit extreme arbitrariness into modal space, hence there is no reason why we should reject one over the other. Moreover, flat-footedly rejecting both seems gratuitous.

What, then, should one conclude from the paradox? Fine [4, p. 224] entertains the idea of rejecting $(\mathrm{R} 2) /(\mathrm{R} 2 *)$ by imposing a set-theoretic limitation of size constraint on the domains of possible worlds. But since we need not understand the domains of worlds set-theoretically, this constraint is difficult to motivate once again for reasons of arbitrariness. For why would it not be possible that there are more than set many things? Moreover, Fine's proposal is not obviously well-motivated for modal primitivists who reject an extensional reduction of modal discourse to possible worlds talk. In contrast to Fine, Fritz is more pessimistic; he [8, p. 8] takes the "puzzle [to] at least chip away at the support for the assumption that the notion of metaphysical modality is in good standing", for the assumption that "we have succeeded in singling out a particular notion of metaphysical necessity" is mostly "a dogma of current metaphysics which is supported by the hope that some core theoretical roles of 
metaphysical necessity [e.g. recombinatorial principles] suffice pick out a particular distinguished modality.",

In what follows, however, it is argued that the prospects are not so bleak for advocates of metaphysical modality. Instead, a novel conception of metaphysical modality is offered which does justice to the theoretical reasons motivating $(\mathrm{R} 1) /(\mathrm{R} 1 *)$ and $(\mathrm{R} 2) /(\mathrm{R} 2 *)$ without lapsing into paradox.

\section{Modal Expansionism}

\subsection{Indefinite Extensibility}

As a crude gloss, a linguistic expression is indefinitely extensible when given all of its instances one can define, by sole reference to those instances, a new instance of the expression that is not included in the collection of the expression's instances from which it was defined. ${ }^{7}$ Roughly speaking, the idea is that such expressions exhibit a distinctive kind of open-endedness insofar as one can indefinitely expand their domain. As Russell [19, p. 36] explains an early form of the idea:

[T] here are what we may call self-reproductive processes and classes. That is, there are some properties such that, given any class of terms all having such a property, we can always define a new term also having the property in question. Hence we can never collect all the terms having the said property into a whole; because, whenever we hope we have them all, the collection which we have immediately proceeds to generate a new term also having the said property.

The notion of indefinite extensibility is familiar to philosophical discussions of iterative set theory. On the iterative conception of set, from a given universe of sets one can define, by sole reference to the sets in that universe, a new set which is not amongst the sets from which it was defined. For instance, take any universe $V$ of sets. One can define a set which does not belong to $V$-namely, the set of of all non-self membered sets in $V$, for on pain of Russell's paradox it cannot belong to $V$.

Following Linnebo [14, pp. 146-147], we can state this idea more precisely. Thus, consider the following comprehension schema of plural logic: ${ }^{8}$

\section{Plural Comprehension $\exists x x \forall u(u \prec x x \leftrightarrow \phi(u))$}

The schema seems uncontroversial: for a condition $\phi$, of course there are the things that $\phi$. Yet despite its seeming innocence, from Plural Comprehension one can derive a contradiction with the following unrestricted principle of set formation:

\footnotetext{
${ }^{6}$ Fritz [8, pp. 10-11] later considers but does not endorse a solution similar to the one proposed in this paper. Rayo (MS) also develops this idea in an interestingly different way.

${ }^{7}$ See Gabriel Uzquiano [25, p. 147]. Note that non-predicate expressions, such as quantifiers, which strictly speaking lack 'instances', are often taken to be indefinitely extensible. See Fine [5], and the other papers in Rayo and Uzquiano [18].

${ }^{8} \mathrm{We}$ assume that the plural quantifier 'there are some things' is to be read as 'there are zero-or-more things'.
} 
Collapse $\forall x x \exists y \forall u(u \prec x x \leftrightarrow u \in y)$

The derivation is straightforward. Informally, instantiating $\phi$ in Plural Comprehension with ' $\notin u$ ' gives us the $r r$, all and only those sets which are not elements of themselves:

(1) $\forall u(u \prec r r \leftrightarrow u \notin u)$

Yet by Collapse, we can infer that there is an $r$ such that:

(2) $\forall u(u \prec r r \leftrightarrow u \in r)$

And we can use (1) and (2) to conclude:

$$
\forall u(u \in r \leftrightarrow u \notin u)
$$

Yet this generates a contradiction when the universal quantifier is instantiated with $r$ itself.

Given the elegance and power of the comprehension schema, the natural reaction is to reject Collapse. However, it is difficult to provide a principled basis for doing so. For instance, one might be tempted to impose a strict constraint on when pluralities form a set, perhaps pertaining to set-theoretic limitation of size. But as Øystein Linnebo [14, pp. 152-154] and others have emphasised, one can appreciate the arbitrariness of such constraints. In an attempt to avoid such arbitrariness, an alternative is to replace Collapse with a slightly weaker but still well-motivated principle. For whereas Collapse states that for any things there is a set containing all those things, a weaker principle states that for any things there could be a set containing all those things. Yet that there merely could be but is not such a set blocks the above derivation.

This approach is characteristic of advocates of indefinite extensibility. They claim that upon introducing the newly defined set, the set-theoretic universe expands; when such an expansion occurs, the membership predicate comes to range over a strictly larger set-theoretic universe. And there is something natural about this picture. For although we saw above that the set of all sets in $V$ which do not belong to themselves cannot itself belong to $V$, it does of course belong to the power set of $V$ - the next rank in the hierarchy. Thus, in introducing this 'Russell set' and forcing an expansion of the set-theoretic universe, the membership predicate comes to range over the more inclusive power set of $V$. Moreover, one need not think of this picture in constructivist terms. The idea is that the various set-theoretic universes are mind-independent and pre-given. Expansion is not then to be understood in terms of universe construction, but rather in terms of inter-linguistic semantic shift.

For another example, consider the notion of an ordinal number. Due to the BuraliForti paradox, it is tempting to think that 'ordinal' is indefinitely extensible. ${ }^{9}$ A version of this paradox is generated by two principles:

(BF1) The ordinal corresponding to any well-ordering of ordinals is strictly larger than any ordinal in the series.

\footnotetext{
${ }^{9}$ For a recent endorsement of this idea, see Fine [5, p. 22]. Thanks to an anonymous reviewer for emphasising the pertinence of this example, who also notes that the Russell quote cited above follows Russell's discussion of the Burali-Forti paradox.
} 
(BF2) Since the ordinals form a well-ordering, there is an ordinal corresponding to it.

To appreciate the paradox, consider the ordinal specified in (BF2). By (BF1) this ordinal, $\Omega$, is strictly larger than any ordinal in the series mentioned in (BF2). However, $\Omega$ is then distinct from all ordinals in that series, which was meant to include all ordinals. Nevertheless, those who claim that 'ordinal' is indefinitely extensible can avoid this contradiction. On their view, in considering the series of ordinals in (BF2), the interpretation of 'ordinal' expands so as to range over the ordinal corresponding to their well-ordering itself. Thus, post-expansion we are no longer considering the series of all ordinals after all. Similar to the set-theoretic example, this expansion is not to be understood in terms of our constructing some new ordinal, but again in terms of inter-linguistic semantic shift.

Strikingly, the principles (BF1) and (BF2) bear a noticeable resemblance to the two recombinatorial principles in the modal paradox, (R1) and (R2). This suggests that positing indefinite extensibility could be key to avoiding the recombinatorial paradox. For example, consider again (R2), the principle which states that for all possible worlds and the elementary particles at those worlds, there is a further possibility at which all those possible elementary particles are elementary particles. It is natural to view this further possibility as introduced by all antecedent possibilities, in a similar sense to which $\Omega$ was introduced above, and to which the Russell set above was introduced by all sets in $V$. Yet just as - on pain of contradiction-the Russell set could not belong to the universe from which it was defined, this newly introduced possibility cannot be amongst those possibilities from which it was introduced. For by (R1) there is no possibility that contains all possible elementary particles.

The claim is that the utterance of (R2) induces an expansion of modal space; in introducing this further possibility, one reinterprets the modal vocabulary in the language to range over a more inclusive modal space. Therefore, this utterance of (R2) is not true under a univocal interpretation of its modal vocabulary. Although according to some interpretation of the metaphysical modal vocabulary all possible elementary particles are all such that according to some more inclusive interpretation possibly they are all elementary particles, it is not the case that according to a given interpretation possibly all possible elementary particles are all elementary particles according to that very interpretation. Thus no contradiction results and the paradox dissolves: the moral is that we must appreciate the disastrous consequences of attempting to interpret the modal vocabulary in one of (R1) or (R2) according to a single modal space. $^{10}$

It is worth emphasising that if the order of the premises were changed so that we read (R2) before (R1), we might read the modal vocabulary in (R2) univocally and the modal vocabulary in (R1) non-univocally. On that presentation of the paradox,

\footnotetext{
${ }^{10}$ There are interesting questions about the metasemantic mechanisms by which utterances cause shifts in interpretation which are beyond the scope of this paper. For discussion, see Studd [23, chap. 8] and Warren $[26]$.
} 
the utterance of (R1) would induce an expansion of modal space. For the utterance of (R2) would introduce a world $w$ such that for all possible worlds $u$ all elementary particles at $u$ are elementary particles at $w$. Yet the utterance of (R1) would then introduce a further possibility, a world where all elementary particles at $w$ are elementary particles and there is something distinct from every elementary particle at $w$ which is also a elementary particle.

Call the view under consideration modal expansionism. According to the expansionist, 'metaphysical necessity' and its interdefinable notions are indefinitely extensible. They maintain that the background modal space over which we modally generalise can always be expanded, under specific contextual circumstances. Moreover, given the indefinite extensibility of 'metaphysical necessity', there will never be an ultimate interpretation of metaphysical modal operators. For any modal space over which such operators range could always be expanded via the introduction of a new possibility. Accordingly, there is one sense in which metaphysical modality is not the maximal member of the family of objective modalities: the objective modalities can always be expanded to include broader notions of necessity. ${ }^{11}$ Nevertheless, it is crucial to emphasise that there are multiple versions of this maximality thesis in the expansionist setting. For instance, the expansionist can still recognise that, holding fixed the interpretation of metaphysical modal vocabulary, metaphysical modality is the maximal objective modality according to that particular interpretation. Indeed, the model theory given below could be generalised to handle other objective modalities in a manner that would completely respect this claim. The upshot is that there is still an important sense in which metaphysical necessity is to be equated with the maximal objective modality.

Finally, to close this section, we note that reductionists about metaphysical modality will have to locate the indefinite extensibility in the non-modal locutions to which they reduce metaphysical modal notions. For instance, on Lewis' [13] concrete modal realist reduction of metaphysical modal notions, since metaphysical modal operators are purged from total theory in favour of first-order quantifiers over worlds, modal expansionism collapses into indefinite extensibility about first-order quantification. ${ }^{12}$ Nevertheless, outside of the Lewisian setting, modal expansionism and expansionism about first-order quantification are independent proposals.

\subsection{Variations}

In the next section, modal expansionism is developed in further detail. Before we turn to those details, however, it will help to consider a slight variant of the recombinatorial paradox. The benefits of doing so are twofold. First, it will allow the reader to see another application of the expansionist solution, which should aid their understanding of how it functions. Second, it will help to clarify a potential confusion about the expansionist solution.

\footnotetext{
${ }^{11}$ This modal claim is articulated with more care in Section 4.

${ }^{12}$ Fine [5], amongst others, defends the view that first-order quantification is indefinitely extensible.
} 
The relevant variant of the recombinatorial paradox employs irreducible plural quantification. ${ }^{13}$ In this paradox, the background plural logic is assumed to be fairly standard, with plural quantifiers admitting an 'empty' plurality so as to validate the Plural Comprehension schema discussed above. (Given that the logic is a modal plural logic, it validates the schema in the sense that each of the schema's instances are true in each 'world' in each model.) In many respects, this variant is closer to Fine's original version which employs quantification over proper classes, for, as is known from the work of Uzquiano [24] and others, such quantification can be understood in terms of irreducible plural quantification.

Like the initial paradox, the variant paradox is based on two principles:

(PI1) Necessarily, the elementary particles are such that possibly they are elementary particles and there is an elementary particle that is not one of them.

(P12) Possibly, the elementary particles are such that necessarily, every elementary particle is one of them.

The line of reasoning which generates the contradiction should now be familiar. Suppose, as (P12) states, that it is possible that the elementary particles comprise every possible elementary particle. Then, in contrast to (P11), it is not the case that in every possibility the elementary particles are possibly elementary particles and accompanied by another elementary particle. ${ }^{14}$

To avoid this contradiction, the expansionist claims that the utterance of (P12) is not true on a univocal reading of its modal vocabulary. Similar to before, the expansionist will view the possibility in which the elementary particles comprise all possible elementary particles as introduced (in the above sense) from some initial possibilities. Thus, in order to isolate this thought, the modal expansionist can specify their non-univocal reading of this particular utterance of $(\mathrm{Pl} 2)$ :

(P12') According to a given interpretation of the metaphysical modal vocabulary, there is some more inclusive interpretation according to which it is possible that the elementary particles comprise everything which is a possible elementary particle according to the initial interpretation.

Moreover, as with the initial recombinatorial paradox, there is an available nonunivocal reading of (P11) utterances too. On this reading, an utterance of (Pl1) can be understood as follows:

(Pl1') According to a given interpretation of the metaphysical modal vocabulary, it is necessary that the elementary particles are such that there is some more

\footnotetext{
${ }^{13}$ See Fritz [8, Appendix B] for discussion of this version of the paradox. He notes that in the presence of certain background model-theoretic conditions, the claims involved in the plurally formulated paradox are model-theoretically equivalent to their corresponding claims in the initial formulation.

${ }^{14}$ As an aside, notice that this version of the paradox makes no use of the Vlach devices. Some may take this to be an advantage, since it is at least contentious whether Vlach operators are legitimate resources for modal primitivists to employ; see, for instance, the exchange between Melia [15] and Forbes [6]. Timothy Williamson [27, pp. 333-334] also questions whether Vlach operators can be legitimately used by contingentists.
} 
inclusive interpretation according to which it is possible that they are all elementary particles and there is an elementary particle that is not one of them.

Thus, although this paradox differs slightly from the initial one, the expansionist offers a similar solution to both: each paradox is to be avoided by recognising the non-univocal readings of the relevant principles.

An interesting question concerning this variant paradox is whether it could be avoided by rejecting Plural Comprehension. If this schema is rejected, at some worlds there may simply be no things, the elementary particles. And, given that the plural logic admits an 'empty' plurality, this is different from there being a world at which there are the zero-many elementary particles, to which nothing belongs. Instead, the claim would involve the radical idea that there could be an individual elementary particle $e_{1}$, an individual elementary particle $e_{2}$, and so on, but no things the elementary particles. On a natural way of treating plural definite descriptions, this will allow $(\mathrm{P} 12)$ to be trivially true, which could provide a means of avoiding the paradox. ${ }^{15}$

Stephen Yablo [29, pp. 151-152] has advocated rejecting Plural Comprehension as a way of capturing the indefinitely extensibility of 'set'. On his view, this indefinite extensibility results from a lack of determinacy in which sets there are. In turn, this lack of determinacy falsifies the relevant instance of the comprehension schema: that there these things, the sets. Nevertheless, regardless of whether this is viable in the set-theoretic case, it should be uncontroversial that it is not indefinitely extensible what elementary particles there are. As Fritz [8, p. 551-552] stresses, entities like elementary particles simply do not exhibit the type of iterative behaviour required for indefinite extensibility. Thus, there is no motivation to reject the instances of the comprehension schema that are relevant to the above recombinatorial paradox.

As a minor aside, notice that Yablo's proposal is different to the above view of the indefinite extensibility of 'set' on which Collapse was rejected over Plural Comprehension. The rejection of Collapse instead of Plural Comprehension is advocated by Linnebo [14, pp. 156-158]. However, Linnebo accepts the previously mentioned modalised version of Collapse and rejects a suitably modalised version of Plural Comprehension. Due to this, Yablo and Linnebo's approaches to the indefinite extensibility of 'set' are very similar. It is worth noting that modal expansionism is naturally coupled with Linnebo's rejection of the modalised version of Plural Comprehension. The following section will specify an interpretation of the modality used in these modalised principles.

This overall point highlights one crucial feature of the expansionist solution. Specifically, it shows that the expansionist is not claiming that at each world the domain of elementary particles is open to expansion. Again, that idea must be accompanied with the implausible thought that the elementary particles exhibit indefinite extensibility. Instead, the expansionist claim is that due to the indefinite extensibility of 'metaphysically necessary', it is indefinitely extensible what metaphysically

\footnotetext{
${ }^{15}$ See Fritz [8, Appendix B] for the semantic details of this point. Roughly put, his gloss of 'the elementary particles are $\psi$ ' is 'for all things, if anything is one of them iff it is an elementary particle, then $\psi$ '.
} 
possible elementary particles there are. This latter idea will be developed carefully and implemented in the following section.

\section{Model Theory}

The expansionist conception of metaphysical modality offers an elegant solution to the recombinatorial paradox. But how exactly should we understand the expansionist's modal claim that we can define some new possibility? Recall that such modal talk was used to explicate the indefinite extensibility of 'set' too. But in the set-theoretic case, it would have been inappropriate to read the modal claims as expressing metaphysical modality, for it is metaphysically necessary which sets there are. Similarly, the modal expansionist should not explicate the claim that we can define some new metaphysical possibility in terms of metaphysical modality either. For if, as is plausible, $\mathbf{S 4}$ is a lower bound on the logic of metaphysical necessity, it is not metaphysically possible that there is some metaphysical possibility which is not metaphysically possible. So how should this new modality be understood?

Given that expansions occur when the metaphysical modal vocabulary is reinterpreted, a natural understanding of the new modality is as an interpretational one. ${ }^{16}$ On this view, the modality concerns possibilities for admissibly reinterpreting the metaphysical modal vocabulary. To be possible is to be true according some such interpretation, to be necessary is to be true according to all of them. And by an admissible reinterpretation of the metaphysical modal vocabulary we mean one which respects the postulates of metaphysical modality's theoretical role and conforms to the axioms of its logic. ${ }^{17}$

In order to study how interpretational and metaphysical modality interact, we can enrich the vocabulary of our quantified modal language with a new modal operator $\mathbf{Q}_{\geq}$(letting $\downarrow_{\geq}$abbreviate $\neg \mathbf{Q}_{\geq} \neg$ ) expressing this particular species of interpretational necessity. We also introduce analogues $\left(\uparrow^{*}, \downarrow^{*}\right)$ of the infinitely many distinct pairs of Vlach-operators we saw previously to achieve cross-reference across reinterpretations. To be precise, let $\mathcal{L}_{2}$ be the language which has the exact vocabulary of $\mathcal{L}_{1}$ plus $\boldsymbol{\square}_{\geq}$and infinitely many distinct pairs of Vlach-operators $\uparrow^{*}$ and $\downarrow^{*}$.

To study the bimodal logic of interpretational necessity and metaphysical necessity, it will help to proceed model-theoretically; this model theory need only be treated instrumentally, since it serves the merely mathematical purpose of providing a consequence relation for the logic. To define an appropriate class of models, we first define a frame $\mathfrak{F}$ as a quadruple $\left\langle\mathcal{I}, \mathcal{R}, \mathcal{W}^{*}, \mathcal{D}\right\rangle$, where $\mathcal{I}$ is a non-empty set understood as a set of admissible interpretations of the metaphysical modal operators of a

\footnotetext{
${ }^{16}$ Uzquiano [25] adopts this understanding of the modality used to explicate indefinite extensibility in set theory, and Studd [22, p. 706] at least entertains it. With this being said, advocates of modal approaches to indefinite extensibility disagree on how to interpret the modality; see Fine [5] and Linnebo [14], for alternative readings of the modal.

${ }^{17}$ For simplicity, we shall assume that the logic of metaphysical necessity is $\mathbf{S 5}$. In the model theory, the definition of the accessibility relations for metaphysical necessity could be altered to validate a weaker logic. See Salmon [20] for an argument that the logic of metaphysical necessity is weaker than S4.
} 
language, and $\mathcal{R}$ is a binary relation on $\mathcal{I}$, understood as an accessibility relation over interpretations of the metaphysical modal vocabulary. Informally, when $i \mathcal{R} j$ one can think of $j$ as an interpretation of the metaphysical modal vocabulary at least as inclusive as $i$, in the sense that all metaphysical possibilities recognised by $i$, and perhaps more, are recognised as metaphysical possibilities by $j$. Model-theoretically, this is encoded by $\mathcal{W}^{*}$, which is a total function from $i \in \mathcal{I}$ to a non-empty set $\mathcal{W}_{i}^{*}$, thought of as the set of metaphysically possible worlds according to $i$. We then define:

$$
\mathcal{W}=\bigcup_{i \in \mathcal{I}} \mathcal{W}_{i}^{*}
$$

Accordingly, $\mathcal{D}$ is a function taking each $w \in \mathcal{W}$, into a non-empty set $\mathcal{D}_{w}$, understood as that metaphysically possible world's domain. We impose the following conditions on frames, the reasons for which will become clear in what is to come:

Transitivity $\quad(\forall i \in \mathcal{I})(\forall j \in \mathcal{I})(\forall l \in \mathcal{I})(i \mathcal{R} j \wedge j \mathcal{R} l \rightarrow i \mathcal{R} l)$

\section{Reflexivity $\quad(\forall i \in \mathcal{I})(i \mathcal{R} i)$}

Directedness $\quad(\forall i \in \mathcal{I})(\forall j \in \mathcal{I})(\forall l \in \mathcal{I})(i \mathcal{R} j \wedge i \mathcal{R} l \rightarrow(\exists m \in \mathcal{I})(j \mathcal{R} m \wedge l \mathcal{R} m))$

Monotonicity $(\forall i \in \mathcal{I})(\forall j \in \mathcal{I})\left(i \mathcal{R} j \rightarrow \mathcal{W}_{i}^{*} \subseteq \mathcal{W}_{j}^{*}\right)$

Finally, with each $i \in \mathcal{I}$ we associate an equivalence relation:

$$
E_{i}=\left\{\langle u, v\rangle: u, v \in \mathcal{W}_{i}^{*}\right\} \cup\{\langle u, u\rangle: u \in \mathcal{W}\}
$$

Each of these relations can be thought of as the accessibility relation over metaphysically possible worlds associated with that interpretation. They are each reflexive over $\mathcal{W}$ in order to ensure that the logic for metaphysical necessity is $\mathbf{S 5}$. Note that given Monotonicity, if $i \mathcal{R} j$ then $E_{i} \subseteq E_{j}$.

An $\mathcal{L}_{2}$ model $\mathfrak{M}$ based on $\mathfrak{F}=\left\langle\mathcal{I}, \mathcal{R}, \mathcal{W}^{*}, \mathcal{D}\right\rangle$ is a quintuple $\left\langle\mathcal{I}, \mathcal{R}, \mathcal{W}^{*}, \mathcal{D}, \mathcal{V}\right\rangle$, where $\mathcal{V}$ is a function taking any individual constant of the language into a member of $\cup_{w \in \mathcal{W}} \mathcal{D}_{w}$, and any $n$-place predicate letter of the language, $i \in \mathcal{I}$, and $u \in \mathcal{W}$, into a set of $n$-tuples of $\cup_{w \in \mathcal{W}} \mathcal{D}_{w}$, that predicate's extension at that world at interpretation. Notice that $\mathcal{V}$ takes any 0 -place predicate letter, i.e. an atomic sentence, $i \in \mathcal{I}$, and $u \in \mathcal{W}$ into a set of 0 -tuples; in this particular case, we think of $\{\langle\rangle\}$ (' $T$ ') as truth and \{\} (' $F$ ') as falsity. $\mathcal{V}$ is also subject to the following constraint (where $\phi$ is an $\mathcal{L}_{2}$ atomic formula):

Stability $\quad(\forall i \in \mathcal{I})(\forall w \in \mathcal{W})(\forall j \in \mathcal{I})(i \mathcal{R} j \rightarrow \mathcal{V}(\phi, i, w)=\mathcal{V}(\phi, j, w))$

Given the below clauses for the connectives and quantifier, this condition entails that any $\mathcal{L}_{2}$ formula which contains no occurrences of any modal operators exhibits the same kind of stability.

An assignment $g$ to the variables in $\mathfrak{M}$ is a function which assigns to each individual variable a member of $\cup_{w \in \mathcal{W}} \mathcal{D}_{w}$. Where $x$ is a variable, two assignments $g$ and $g^{\prime}$ are $x$-alternatives iff $g$ and $g^{\prime}$ assign the same values for all variables, except possibly $x$. 
We define ' $(\mathfrak{M}, \overline{\text { seq }}, i$, seq, $w) \models_{g} A^{\prime}$ for $\mathfrak{M}=\left\langle\mathcal{I}, \mathcal{R}, \mathcal{W}^{*}, \mathcal{D}, \mathcal{V}\right\rangle, \overline{\text { seq }} \in \mathcal{I}^{<\omega}$, $i \in \mathcal{I}$, seq $\in \mathcal{W}^{<\omega}, w \in \mathcal{W}, g$ an assignment to the variables in $\mathfrak{M}$, and $A$ a formula of the language, recursively as follows:

$(\mathfrak{M}, \overline{\text { seq }}, i, s e q, w) \models_{g} F a_{1} \ldots a_{n}$ iff $\left\langle\mathcal{V}_{g}\left(a_{1}\right), \ldots, \mathcal{V}_{g}\left(a_{n}\right)\right\rangle \in \mathcal{V}(F, i, w)$;

$(\mathfrak{M}, \overline{s e q}, i, s e q, w) \models_{g} \neg A$ iff $(\mathfrak{M}, \overline{s e q}, i, s e q, w) \not \models_{g} A$;

$(\mathfrak{M}, \overline{s e q}, i, s e q, w) \models_{g} A \rightarrow B$ iff either $(\mathfrak{M}, \overline{s e q}, i$, seq,$w) \not \models_{g} A$ or $(\mathfrak{M}, \overline{s e q}, i, s e q, w) \models_{g} B$;

$(\mathfrak{M}, \overline{\text { seq }}, i$, seq,$w) \models_{g} \forall x A$ iff $(\mathfrak{M}, \overline{s e q}, i$, seq,$w) \models_{g^{\prime}} A$ for every $x$-alternative $g^{\prime}$ of $g$, such that $g^{\prime}(x) \in \mathcal{D}_{w}$;

$(\mathfrak{M}, \overline{\text { seq }}, i$, seq,$w) \models_{g} \square A$ iff for every $u$ such that $w E_{i} u$, $(\mathfrak{M}, \overline{s e q}, i, \operatorname{seq}, u) \models_{g} A$

$(\mathfrak{M}, \overline{\operatorname{seq}}, i, \operatorname{seq}, w) \models_{g} \uparrow^{n} A$ iff $\left(\mathfrak{M}, \overline{\operatorname{seq}}, i, \operatorname{seq}^{n \rightarrow w}, w\right) \models_{g} A$;

$(\mathfrak{M}, \overline{\operatorname{seq}}, i, \operatorname{seq}, w) \models_{g} \downarrow^{n} A$ iff $(\mathfrak{M}, \overline{\operatorname{seq}}, i, \operatorname{seq}, \operatorname{seq}(n)) \models_{g} A$;

$(\mathfrak{M}, \overline{\text { seq }}, i$, seq,$w) \models_{g} \mathbf{Q}_{\geq} A$ iff for every $j \in \mathcal{I}$ such that $i \mathcal{R} j$, $(\mathfrak{M}, \overline{s e q}, j, \operatorname{seq}, w) \models_{g} A$;

$(\mathfrak{M}, \overline{\operatorname{seq}}, i, \operatorname{seq}, w) \models_{g} \uparrow^{* n} A$ iff $\left(\mathfrak{M}, \overline{\operatorname{seq}}^{n \rightarrow i}, i\right.$, seq,$\left.w\right) \models_{g} A$;

$(\mathfrak{M}, \overline{\operatorname{seq}}, i, \operatorname{seq}, w) \models_{g} \downarrow^{* n} A$ iff $(\mathfrak{M}, \overline{\operatorname{seq}}, \overline{\operatorname{seq}}(n), \operatorname{seq}, w) \models_{g} A$.

A formula $A$ is true at $\langle\overline{\text { seq }}, i, s e q, w\rangle$, for $\overline{\operatorname{seq}} \in \mathcal{I}^{<\omega}, i \in \mathcal{I}$, seq $\in \mathcal{W}^{<\omega}, w \in \mathcal{W}$ iff for all variable assignments $g,(\mathfrak{M}, \overline{\text { seq }}, i, s e q, w) \models_{g} A$. A formula $A$ of $\mathcal{L}_{2}$ is true in an $\mathcal{L}_{2}$ model $\mathfrak{M}=\left\langle\mathcal{I}, \mathcal{R}, \mathcal{W}^{*}, \mathcal{D}, \mathcal{V}\right\rangle$ iff it is true at $\langle\overline{s e q}, i, s e q, w\rangle$, for every $\overline{\text { seq }} \in \mathcal{I}^{<\omega}$, for every $i \in \mathcal{I}$, every seq $\in \mathcal{W}^{<\omega}$, and every $w \in \mathcal{W}$. A formula $A$ of $\mathcal{L}_{2}$ is valid iff it is true in every $\mathcal{L}_{2}$ model. A set $\Gamma$ of $\mathcal{L}_{2}$ formulas entails an $\mathcal{L}_{2}$ formula $A$ iff for every $\mathcal{L}_{2}$ model $\mathfrak{M}=\left\langle\mathcal{I}, \mathcal{R}, \mathcal{W}^{*}, \mathcal{D}, \mathcal{V}\right\rangle$, for every $\overline{\operatorname{seq}} \in \mathcal{I}^{<\omega}$, every $i \in \mathcal{I}$, every seq $\in \mathcal{W}^{<\omega}$, every $w \in \mathcal{W}$ and every variable assignment $g$, if $(\mathfrak{M}, \overline{\text { seq }}, i$, seq,$w) \models_{g} \gamma$ for each $\gamma \in \Gamma$, then $(\mathfrak{M}, \overline{s e q}, i, s e q, w) \models_{g}$ A. Again, for convenience, we shall talk of some $\mathcal{L}_{2}$ formulas (as opposed to their set) entailing another $\mathcal{L}_{2}$ formula.

An S4.2 axiom system is sound and complete for the class of models in which the accessibility relation is a directed partial order. Thus Transitivity, Reflexivity, and Directedness ensure that $\mathbf{Q}_{\geq}$obeys the following axiom schemas, the addition of all instances of which to the minimal normal modal logic yields system S4.2:

(T) $\mathbf{\square}_{\geq} \phi \rightarrow \phi$

(4) $\mathbf{\square}_{\geq} \phi \rightarrow \mathbf{a}_{\geq} \mathbf{a}_{\geq} \phi$

(G) $\boldsymbol{\nabla}_{\geq} \phi \rightarrow \mathbf{\square}_{\geq} \boldsymbol{\nabla}_{\geq} \phi$

It is helpful to notice that the subset relation on the power set of a set is also a directed partial order, since for any members of the power set $s_{1}, s_{2}, s_{3}$, if $s_{1} \subseteq s_{2}$ and $s_{1} \subseteq s_{3}$ then there is some member $s_{4}$ of the power set such that $s_{2} \subseteq s_{4}$ and $s_{3} \subseteq s_{4}$, namely the union of $s_{2}$ and $s_{3}$. This neatly parallels the idea that the set of metaphysically possible worlds according to an interpretation is a subset of the set of metaphysically possible worlds according to any at least as inclusive interpretation, and thus lends credibility to the above conditions on $\mathcal{R}$. Note however that the analogy is imperfect insofar as the subset relation on the power set of a set has a maximal element. 
Axiom schema (4) is equivalent to $\left.\boldsymbol{}_{\geq} \phi \rightarrow\right\rangle_{\geq}$. The Transitivity condition which validates all instances of the axiom schema thus corresponds to the thought that in moving through interpretations of the metaphysical modal operators, no previously inadmissible meaning assignment becomes admissible. Of course, previously admissible ones will no longer be in the range of $\boldsymbol{n}_{\geq}$as one moves through this process, since they will not be at least as inclusive as the interpretation to which one has moved, hence why the accessibility relation is not symmetric.

James Studd [22, p. 703], one advocate of a modal approach to the indefinite extensibility of set-theoretic expressions, requires the following frame condition which entails Directedness.

Connectedness $(\forall i \in \mathcal{I})(\forall j \in \mathcal{I})(\forall l \in \mathcal{I})(i \mathcal{R} j \wedge i \mathcal{R} l \rightarrow j \mathcal{R} l \vee l \mathcal{R} j)$

In the current setting, this has the effect of validating all instances of the following schema:

$$
\mathbf{\square}_{\geq}\left(\mathbf{\square}_{\geq} \phi \rightarrow \psi\right) \vee \mathbf{\square}_{\geq}\left(\mathbf{\square}_{\geq} \psi \rightarrow \phi\right)
$$

However, it would be too restrictive to impose Connectedness for current purposes. To see why informally, suppose that at interpretation $i$ there are $k$ metaphysically possible elementary particles and $k$ metaphysically possible spacetime points. One could run through the initial recombinatorial puzzle with which we began to shift to an interpretation, $j$, at which there are $m$ (for $m>k$ ) metaphysically possible elementary particles and $k$ metaphysically possible spacetime points. ${ }^{18}$ But, at $i$, one could also run through a version of the initial recombinatorial puzzle in which all instances of 'elementary particle' are replaced with 'spacetime point' to shift to an interpretation, $l$, at which there are $m$ metaphysically possible spacetime points and $k$ metaphysically possible elementary particles. But it is not the case that $j$ is at least as inclusive as $l$, or vice versa. The pair thus serves as a counterexample to Connectedness.

Imposing the Stability condition validates the following schema, where $\phi$ is a formula of the language containing no occurrences of any modal operator:

$$
\mathbf{u}_{\geq} \phi \vee \mathbf{\square}_{\geq} \neg \phi
$$

This is desirable, since what is non-modally the case at a world should not be affected by the expansion of metaphysical modal space. Additionally, Monotonicity has the effect of validating a bimodal converse Barcan schema:

(BCBF) $\boldsymbol{\square}_{\geq} \square \forall v \phi \rightarrow \forall v \boldsymbol{\square}_{\geq} \square \phi$

In other terms, it has the effect of ensuring that if according to all at least as inclusive interpretations of the metaphysical modal vocabulary it is metaphysically necessary that everything $\phi \mathrm{s}$, then everything is such that according to all at least as inclusive interpretations it is metaphysically necessary that it $\phi \mathrm{s}$. In slogan form: the possibilia may increase, but none are left behind.

\footnotetext{
${ }^{18}$ Of course, this makes the highly plausible assumption that the broad recombinatorial reasoning employed previously guarantees there will be a more inclusive interpretation according to which there are additional metaphysically possible elementary particles despite there being no additional metaphysically possible spacetime points (and vice-versa).
} 
It is natural to enquire about the status of other bimodal principles. One might first wonder whether the modalities commute. The following principle is $\mathcal{L}_{2}$-valid:

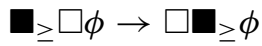

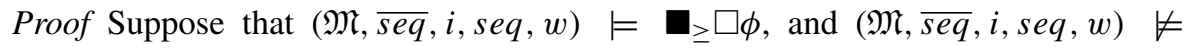
$\square \mathbf{a}_{\geq} \phi$. From the second conjunct, it follows that (M, $\left.\overline{\operatorname{seq}}, i, \operatorname{seq}, u_{2}\right) \forall \mathbf{\square}_{\geq} \phi$ for some $u_{2}$ such that $w E_{i} u_{2}$. Therefore (M, $\overline{\mathrm{seq}}, j$, seq, $\left.u_{2}\right) \not \models \phi$ for some $j \in \mathcal{I}$ such that $i \mathcal{R} j$. But from the first conjunct, it follows that (M, $\overline{\operatorname{seq}}, l, s e q, w) \models \square \phi$ for all $l \in \mathcal{I}$ such that $i \mathcal{R} l$. This includes $j$, so $(\mathfrak{M}, \overline{s e q}, j, s e q, w) \models \square \phi$. It then follows that $\left(\mathfrak{M}, \overline{s e q}, j, s e q, u_{1}\right) \models \phi$ for all $u_{1}$ such that $w E_{j} u_{1}$. Yet $w E_{j} u_{2}$ because $E_{i} \subseteq E_{j}$ and $w E_{i} u_{2}$. Contradiction.

Plausibly, this is the right result: if for any way of more liberally interpreting metaphysical modal operators it is metaphysically necessary that $\phi$, then it is metaphysically necessary that for any way of more liberally interpreting metaphysical modal operators, $\phi$. In part, this reflects the fact that the admissible interpretations do not change from metaphysically possible world to world when the interpretation is held fixed. However, (C1)'s converse is not $\mathcal{L}_{2}$-valid:

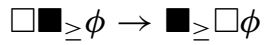

Proof Let $\mathfrak{M}$ be an $\mathcal{L}_{2}$ model such that:

$$
\begin{aligned}
& \mathcal{I}=\{0,1\} \\
& \mathcal{R}=\{\langle 0,1\rangle,\langle 0,0\rangle,\langle 1,1\rangle\} \\
& \mathcal{W}_{0}^{*}=\{2\} \\
& \mathcal{W}_{1}^{*}=\{2,3\} \\
& \mathcal{V}(\phi, i, w)= \begin{cases}T, & \forall i \in \mathcal{I}, \text { if } \phi=A, w=2 \\
F, & \text { otherwise }\end{cases}
\end{aligned}
$$

for $\phi$ an atomic sentence of $\mathcal{L}_{2}$. For atomic 'A', ( $\left.\mathfrak{M}, \overline{s e q}, 0, s e q, 2\right) \models A$, $(\mathfrak{M}, \overline{s e q}, 1, s e q, 2) \models A$ and $(\mathfrak{M}, \overline{s e q}, 1, s e q, 3) \not \models A$, for arbitrary $\overline{s e q}$ and seq. It is easily checked that (M, $\overline{\mathrm{seq}}, 0$, seq, 2) $\not \models \square \mathbf{u}_{\geq} A \rightarrow \mathbf{\square}_{\geq} \square A$.

For an informal counterexample to (C2), suppose that at $i$ there is no metaphysically possible world at which there are at least $k$ elementary particles. Let ' $A$ ' be the nonmodal sentence that there are fewer than $k$ elementary particles. At $i, \square \mathbf{a}_{\geq} A$, for since ' $A$ ' contains no modal vocabulary the $\mathbf{\square}_{\geq}$operator is redundant, yet $\square A$ since at $i$ it is metaphysically necessary that there are fewer than $k$ elementary particles. However, at $i$ it is not the case that $\boldsymbol{\square}_{\geq} \square A$. For according to some more inclusive interpretation of the language's metaphysical modal terms, there is a metaphysically possible world that contains more than $k$ elementary particles. So at $i$ it is false that 
according to every at least as inclusive interpretation of the language's metaphysical modal terms there is a metaphysical modal maximum of $k$ elementary particles.

It is straightforward to check that the principles $\square \phi \rightarrow \boldsymbol{\square}_{>} \phi$ and $\boldsymbol{\square}_{>} \phi \rightarrow \square \phi$ fail too. Yet despite neither modality implying the other, certain bimodal formulae of $\mathcal{L}_{2}$ are of distinctive theoretical interest, such as those concerning which metaphysical modal facts hold according to all interpretations. For example, as noted above, since each $E_{i}$ is an equivalence relation, at every interpretation the theorems of $\mathbf{S 5}$ hold for $\square$. Thus suitably interpretationally-modalized versions of the theorems of $\mathbf{S 5}$ for $\square$ will also hold. Such formulae will concern the metaphysical modal facts whatever the interpretation of the metaphysical modal operators, not merely the metaphysical modal facts according to some particular interpretation. ${ }^{19}$ But note that merely prefixing a $\boldsymbol{\square}_{\geq}$operator to each theorem of $\mathbf{S 5}$ will not suffice to express such facts. For the resulting formula would concern only all at least as inclusive admissible interpretations of the metaphysical modal operators, and not all such interpretations. Thus we can introduce into the language an operator $\mathbf{\square}$ as follows, where $R^{+}$is the transitive closure of $R \cup R^{-1}$ :

$$
(\mathcal{M}, \overline{s e q}, i, \operatorname{seq}, w) \models \mathbf{\square} \text { iff for each } j \in \mathcal{I} \text { such that } i R^{+} j,
$$

$$
(\mathcal{M}, \overline{s e q}, j, s e q, w) \models A
$$

$R^{+}$is an equivalence relation, so $\mathbf{D}$ obeys an $\mathbf{S 5}$ modal logic.

The operator allows one to state absolutely general truths about metaphysical modal facts whatever the interpretation of the metaphysical modal operators. For example, take an instance of the $\mathbf{S 5}$ axiom schema:

(S51) $\diamond A \rightarrow \square \diamond A$

This is -modalized as follows:

$\left(\mathrm{S}_{2}\right) \quad \square(\diamond A \rightarrow \square \diamond A)$

(S52) is valid, since $\boldsymbol{\square}$ conforms to the rule of necessitation (if $\models \phi$ then $\models \boldsymbol{Q}$ ), and as previously mentioned $\left(\mathrm{S}_{1}\right)$ is valid since each $E_{i}$ is an equivalence relation. Thus, at any interpretation, object-language principles prefixed with $\mathbf{\square}$ allow us to state absolutely general truths about the structure of metaphysical necessity at every interpretation.

A simple, but crucial, point to notice is that the model theory allows for the extension of a predicate such as 'metaphysically possible elementary particle' at a particular metaphysically possible world to vary from interpretation to interpretation; specifically, it may expand as one shifts to a more inclusive interpretation. ${ }^{20}$ Indeed, we can utilise this feature to formalise an analogue of $(\mathrm{R} 2 *)$ which is true, speaks to

\footnotetext{
${ }^{19}$ Compare Uzquiano [25, p. 155] on modalisations of the axioms of set theory.

${ }^{20}$ Strictly speaking, the formal language does not contain complex predicates like 'metaphysically possible elementary particle', but one can think of this point in terms of the satisfaction of open formulae.
} 
the concerns of not imposing arbitrariness which motivate (R2), and from which no paradox results: ${ }^{21}$

$(\mathbf{R} 2)$

$$
\uparrow^{* 1} \uparrow^{1} \succ \uparrow^{* 2} \diamond \uparrow^{2} \downarrow^{* 1} \downarrow^{1} \square \forall x\left(E(x) \rightarrow \downarrow^{* 2} \downarrow^{2} E(x)\right)
$$

(更2) says that according to an interpretation, there is some at least as inclusive interpretation of the language such that according to the latter interpretation it is metaphysically possible that everything which is a elementary particle at any metaphysically possible world at the initial interpretation is a elementary particle.

Similarly, with this formal apparatus, one can now formalise an analogue of (R1*) which is true, speaks to the concerns of not imposing arbitrariness with motivate (R1), and from which no paradox results:

$$
\begin{gathered}
\text { (口R1) } \uparrow^{* 1} \square \uparrow^{1} \diamond_{\geq} \uparrow^{2} \diamond \uparrow^{2}\left(\downarrow^{* 1} \downarrow^{1} \forall x\left(E(x) \rightarrow \downarrow^{* 2} \downarrow^{2} E(x)\right) \wedge\right. \\
\left.\exists x\left(E(x) \wedge \downarrow^{* 1} \downarrow^{1} \neg E(x)\right)\right)
\end{gathered}
$$

( $\mathrm{R} 1$ ) says that according to an interpretation there is some at least as inclusive interpretation at which it is metaphysically possible that everything which is a elementary particle at any metaphysically possible world at the initial interpretation is a elementary particle, and there is some elementary particle which is not a elementary particle at any metaphysically possible world at the initial interpretation.

Moreover, unlike (R1*) and (R2*), the replacement principles $(\boldsymbol{\nabla} 1)$ and $(\boldsymbol{\nabla} 2)$ are satisfiable, in the sense that we can construct $\mathcal{L}_{2}$ models in which they are both true. One class of such models are those which reflect the informal idea that at each interpretation there is a world $w$ which contains all possible elementary particles at that interpretation, but at a more inclusive interpretation there is an additional world $u$ which contains all elementary particles at $w$ and a elementary particle distinct from all those at $w$ :

Let $\mathfrak{M}$ be an $\mathcal{L}_{2}$-model such that:

$$
\begin{aligned}
& \mathcal{I}=\{x: x \text { is an odd natural number }\} \\
& \mathcal{R}=\{\langle x, y\rangle: x \text { is less than or equal to }\} \\
& \mathcal{W}_{i}^{*}=\{x<i: x \text { is an even natural number }\} \\
& \left.\mathcal{D}_{w}=\{1 / n: n \text { a non-zero even natural number }\} \text { (for all } w \in \mathcal{W}\right) \\
& \mathcal{V}(\phi, i, w)= \begin{cases}\{1 / 2, \ldots, 1 / w+2\}, & \text { when } \phi=G \text { and } i>w \\
\{\}, & \text { otherwise }\end{cases}
\end{aligned}
$$

For ease of presentation, let us label $1 / \mathrm{n}$ ' $m_{n-2}$ ', so that $1 / 2$ is ' $m_{0}$ ', $1 / 4$ is ' $m_{2}$ ' and so on. We can then depict the model as follows:

\footnotetext{
${ }^{21}$ The advocate of indefinitely extensible metaphysical modality can maintain that (R2)-a sentence of English-is implicitly interpretational modal and thus true. But since the formal quantified modal language of which $(\mathrm{R} 2 *)$ was a formula did not contain interpretational modal operators (R2*) cannot be implicitly interpretationally modal, and is therefore false.
} 


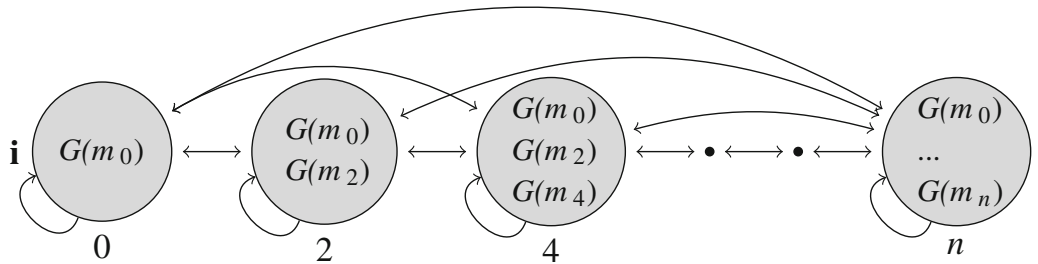

-
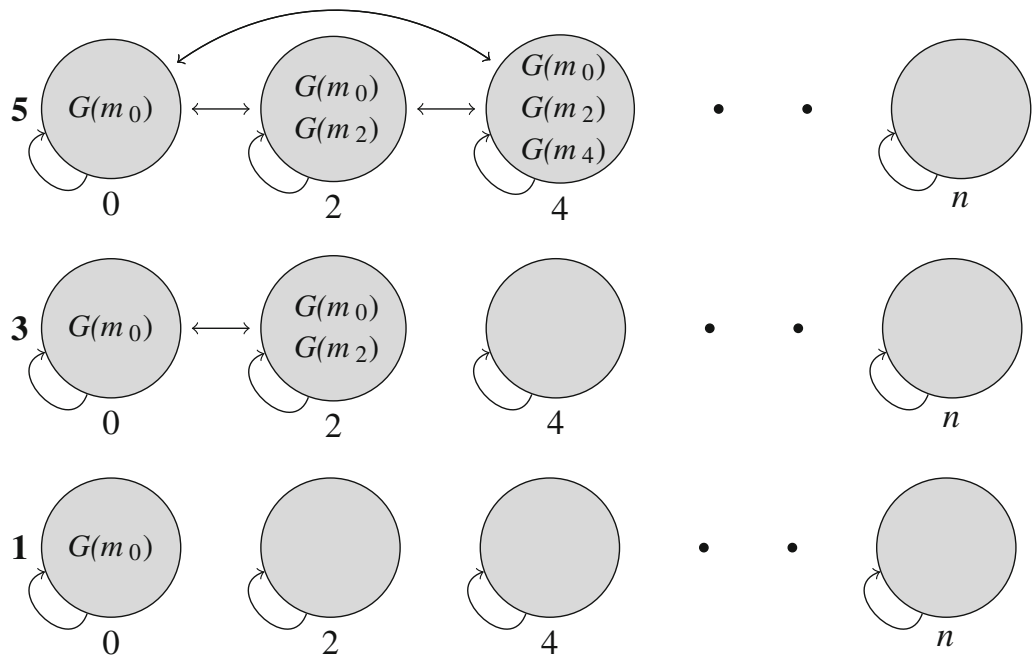

One can check that $(\boldsymbol{\nabla} 1)$ and $(\boldsymbol{\square} 2)$ are both true in $\mathfrak{M}$.

\section{Revenge}

One might wonder whether the modality expressed via the string of modal operators $\square$ can supplant metaphysical necessity in various respects. However, this issue is not at all straightforward: if the modality $\square \square$ is too similar to (our previous conception of) metaphysical necessity, revenge paradoxes threaten. In this section, we explore this issue and the various ways in which $\square \square$ might differ from (our previous conception of) metaphysical necessity.

It will help first to consider a similar recombinatorial paradox against the modality expressed by $\square \square$. Call this 'necessity*', and $\diamond$ 'possibility*', and consider the following argument:

(R1') For any possible* world $w$, there is a possible* world $u$ where all elementary particles at $w$ are elementary particles and there is something distinct from every elementary particle at $w$ which is also a elementary particle. 
$\left(\mathbf{R 2}^{\prime}\right)$ There is some possible* world $w$ such that for all possible* worlds $u$ all elementary particles at $u$ are elementary particles at $w$.

Yet considering $w$ in $\left(\mathrm{R} 2^{\prime}\right)$, from $\left(\mathrm{R} 1^{\prime}\right)$ we can infer that:

(C') There is a possible* world $u$ where all elementary particles at $w$ are elementary particles and there is also a elementary particle who is not identical with any of them.

But all the elementary particles at $w$ are all the elementary particles at all possible* worlds. Contradiction. Is this argument sound?

$\left(\mathrm{R} 1^{\prime}\right)$ is licensed by the modal expansionist solution to the initial recombinatorial paradox. For according to any interpretation there is some more inclusive interpretation according to which for every metaphysically possible world $w$ at the previous interpretation, there is a metaphysically possible world at which every elementary particle at $w$ is a elementary particle and there is a elementary particle distinct from any elementary particle at $w$.

However, why believe $\left(\mathrm{R} 2^{\prime}\right)$ ? That is, why believe that at an interpretation there is some metaphysically possible world at which all possible* elementary particles-all elementary particles at any metaphysically possible world at any interpretationare elementary particles? This claim seems antithetical to modal expansionism. For modal expansionists maintain that given any metaphysical modal space one can shift the interpretation to a more inclusive space which contains additional metaphysical possibilities. Such possibilities will include the possibility of there being all possible elementary particles in the initial modal space accompanied by an extra elementary particle. Yet if $\left(\mathrm{R} 2^{\prime}\right)$ were true, then at some interpretation $i$, it would be metaphysically possible that all possible* elementary particles - that is, all metaphysically possible elementary particles according to all interpretations - are elementary particles. But the modal expansionist's point throughout has been that at an interpretation $i$ not everything which is a metaphysically possible $\phi$ according to some more inclusive interpretation is a metaphysically possible $\phi$ at $i$.

The crucial point is that one should not expect the recombinatorial principles which govern metaphysical modality to have $\square \square$ analogues. In particular, and in contrast to metaphysical modality, the expansionist has introduced nothing to the theoretical role of $\square$ which licenses the thought that one should be able to collect all $\square \square$-possibilities of a certain kind into a single $\square \square$-possibility. Compare indefinite extensibility in the set-theoretic case: there is no interpretation of set-theoretic vocabulary according to which every interpretationally possible set is a set.

Nevertheless, this is a negotiable decision point. If one is concerned that the modal expansionist is guilty of imposing arbitrariness onto $\square \square$-modal space as result of rejecting $\left(\mathrm{R} 2^{\prime}\right)$, one can respond more concessively. For one might be tempted to claim that the modality expressed by $\square$, necessity*, is itself indefinitely extensible. Expansionists of this disposition could extend their treatment of the initial paradox to the revenge version. Of course, this thesis of indefinite extensibility would too have to be explicated modally in terms of a different interpretational modality $\mathbf{\square}^{2}$ (we relabel the original $\boldsymbol{\square}$ as $\mathbf{Q}^{1}$ ). And similar revenge paradoxes would threaten the modality expressed by $\mathbf{\square}^{2} \mathbf{\square}^{1} \square$. Thus the expansionist would have to iterate the idea 
that interpretational modalities are indefinitely extensible. Any paradox involving the modality expressed by $\square^{n}, \boldsymbol{\square}^{n-1}, \ldots, \boldsymbol{\square}^{1} \square$ would be handled by a introducing a new interpretational modality $\mathbf{a}^{n+1}$. Moreover, on natural ways of developing this proposal, further modalities would have to be introduced at limit states in a typical way: $\boldsymbol{\square}^{\omega} \phi \leftrightarrow \bigwedge_{n<\omega} \boldsymbol{\square}^{n} \phi$. In turn, these limit modalities would generate further iterations and other limit modalities. However, since it is plausible that 'ordinal' is itself indefinitely extensible, there will be no modality expressed by $\boldsymbol{\square}^{\alpha} \phi$, for every interpretationally possible ordinal $\alpha{ }^{22}$

On the resultant view, for any interpretation $i$ of $\square \square$ there will be some more inclusive interpretation $j$ of $\square \square$ such that the modality expressed by $\square \square$ at $i$ is strictly less inclusive than the modality expressed by $\square$ at $j$. It is important to emphasise the costs of this response. If the indefinite extensibility of modality is iterated as such, one must forfeit absolute metaphysical modal generality. But if the choice is between imposing arbitrariness on metaphysical modal space and recognising our own expressive limitations, perhaps humility seems preferable to imposition.

\section{Further Application}

Modal expansionism enjoys further benefits. The literature on metaphysical modality contains a wealth of other recombinatorial puzzles and paradoxes which are typically presented as attacks on the notion of metaphysical necessity, or some particular theory thereof. Unlike the recombinatorial paradox considered above, these arguments employ auxiliary machinery such as certain set-theoretic principles or certain conceptions of propositions. In response to these other puzzles and paradoxes, typically philosophers have suggested modifying the auxiliary machinery which they employ. However, these responses are parochial and fail to provide a solution which generalises to other modal recombinatorial paradoxes. In contrast, a benefit of the modal expansionist solution is that it can offer a unified solution to all such paradoxes. This greater generality further motivates the modal expansionist view.

In this section, we shall consider two well-known recombinatorial paradoxes: David Kaplan's [11] 'Kaplan's paradox', and argument due to Daniel Nolan [16] which was later developed by Ted Sider [21]. ${ }^{23}$ We shall see how modal expansionist treats each paradox, and, along the way, consider how the expansionist approach compares to some other extant solutions. The discussion will highlight aspects of the expansionist approach which may have gone unnoticed.

\subsection{Kaplan's Paradox}

Kaplan's paradox runs as follows. Suppose the set of metaphysically possible worlds is of cardinality $k$. According to coarse-grained views of propositions that individuate propositions by a criterion of necessary equivalence, each subset of this set is

\footnotetext{
${ }^{22}$ This final use of 'interpretational possibility' should be understood as invoking the Studd-Linnebo interpretational modality used to explicate the indefinite extensibility of 'set'.

${ }^{23}$ Kaplan [11, p. 41, f.n.1] remarks that he first presented the paradox during the 1970 s.
} 
the proposition true at all and only the metaphysically possible worlds belonging to that subset. Hence by the power set operation, there are $2^{k}$ propositions. But consider a person and a time. For any proposition, it is metaphysically possible that it is entertained as the only content that person entertains at that time. Call this a proposition's being queried. There are therefore distinct metaphysically possible worlds corresponding to the occurrence of each query. It follows that there are at least $2^{k}$ worlds. Contradiction.

Several solutions to the paradox have been offered. Kaplan [11] himself takes the paradox to demonstrate that we ought to adopt a ramified conception of propositions (and spaces of worlds), and David Lewis [13, pp. 107-108] draws the moral that some propositions are not in principle entertainable as mental contents. But, as emphasised previously, whatever the merits of such solutions, they lack generality. Clearly, Kaplan's paradox invokes a recombinatorial principle: the premise that for any proposition determined by a metaphysical modal space there is a metaphysically possible world in that space where that proposition is uniquely entertained. Yet given the alleged paradoxes to which similar recombinatorial premises give rise, it would be sensible to expect this particular principle to do so too.

Indeed, the modal expansionist blames the recombinatorial principle and not the particular theory of propositions or mental content. After all, there would appear to be little reason to complicate one's theory of propositions or mental content if one already recognises the tendency of recombinatorial principles to induce expansion. Thus, the expansionist will offer their familiar diagnosis of the paradox. On their view, the recombinatorial principle about the querying of the propositions which a metaphysical modal space determines is only true when its modal vocabulary is read non-univocally. The true reading in question is as follows:

(KP) According to some interpretation, for any proposition determined by the metaphysical modal space at that interpretation, there is some more inclusive interpretation according to which there is a metaphysically possible world where that proposition is uniquely entertained (i.e. queried).

Thus, given some metaphysical modal space of cardinality $k$, there is some more inclusive metaphysical modal space of cardinality $2^{k}$ at which all propositions determined by the previous metaphysical modal space are metaphysically possibly queried. Yet no contradiction results from this.

At this point, will be fruitful to compare the expansionist approach to Kaplan's ramificational response to the paradox. Although, as mentioned above, Kaplan's approach is less general than expansionism, it raises some interesting aspects of expansionism which previously may not have been salient.

The basic idea behind Kaplan's ramificational approach is that the space of possibilities is partitioned into levels indexed by the natural numbers, and this partition induces a further partition on the space of propositions. As Kaplan [11, p. 45] evocatively describes it:

At level 0, there [are] only the possibilities of, say, different distributions of earth, air, fire, and water. Now [at level 1] there are different distributions of earth, air, fire, and water with it being queried whether all is earth, and there 
are the same distributions with it not being queried whether all is earth. These new possibilities provide new propositions to query-for example, whether it is queried whether it is queried whether all is earth.

On Kaplan's view, then, a level $n$ space of possibilities generates a corresponding level $n$ space of propositions, propositions about level $n$ possibilities. Then, those level $n$ propositions can be queried at level $n+1$ possibilities. Thus, no level $n$ proposition is ever queried at a level $n$ possibility, which allows one to resist the reasoning in Kaplan's paradox.

Clearly, the ramificational approach is similar to expansionist approach. Advocates of the former partition the space of possibilities into successively more inclusive levels, and advocates of the latter claim that any space of possibilities can be expanded to a more inclusive space. More generally, although the exact relation between ramification and indefinite extensibility is vexed, it is natural to view the former as something of a precursor to the latter. (This point is not confined to their applications to metaphysical modality.) However, one must appreciate thatas described by Kaplan - the ramificational approach does not have the capacity to solve the initial recombinatorial puzzle of the elementary particles. For, presumably, matters concerning elementary particles are matters of earth, air, fire, and water, so to speak. And, as Kaplan himself emphasises, no possibilities only about new distributions of earth, air, fire, and water are introduced at level 1. Instead, at level 1, there are the same distributions of earth, air, fire, and water along with various level 0 propositions being either queried or not queried.

With this being said, Kaplan's discussion of the ramificational approach raises an interesting issue for expansionists. For given the similarity of both approaches, one might expect that modal expansionism is naturally coupled with an expansionist conception of propositions, similar to how ramifying the space of possibilities induced a ramification of propositions. ${ }^{24}$ Indeed, if propositions are identified by a criterion of metaphysically necessary equivalence, an expansionist conception of propositions is simply a corollary of modal expansionism. Whether this is borne out on alternative conceptions of propositions deserves further discussion, although here it would take us too far afield.

\subsection{Lewis \& Williamson}

Building on Daniel Nolan's [16] work, Ted Sider [21] has argued that both David Lewis' concrete modal realism and Timothy Williamson's first-order necessitism are incompatible with the Urelement Set Axiom. Once again, a recombinatorial principle features centrally in each respective argument:

Moderate Plenitude For each cardinal number $v$, it is metaphysically possible that there exist $v$ non-sets.

\footnotetext{
${ }^{24} \mathrm{Yu}[30]$ discusses what is naturally classified as an expansionist conception of propositions.
} 
Assuming concrete modal realism, Moderate Plenitude entails that there cannot be a set of all non-sets, thus violating the Urelement Set Axiom. For suppose that there were a set $S$ of all non-sets. Let its cardinality be $k$. Let $v$ be some cardinal number larger than $k$. By Moderate Plenitude it is metaphysically possible that there exist $v$ non-sets. Thus by Lewis' concrete modal realism there is a metaphysically possible world containing $v$ non-sets, all of which are members of $S$. So $S$ 's cardinality is not $k$. Contradiction.

Similarly, first-order necessitism entails that there cannot be a set of all non-sets, again in violation of the Urelement Set Axiom. For suppose that there were a set $S$ of all non-sets. Let its cardinality be $k$. Let $v$ be some cardinal number larger than $k$. By Moderate Plenitude it is metaphysically possible that there exist $v$ non-sets. Since the first-order necessitist holds that everything which metaphysically possibly is something is actually something, by Moderate Plenitude there are actually $v$ nonsets, all of which are members of $S$. (This of course assumes that metaphysically possible non-sets are necessarily as such, if they exist.) So $S$ 's cardinality is not $k$. Contradiction.

It may be helpful to notice that Lewisian concrete modal realists are naturally categorised as first-order necessitists. ${ }^{25}$ For when the quantifiers are read unrestrictedly, the Lewisian concrete modal realist will endorse the necessitist claim that necessarily everything is necessarily something. Moreover, the above recombinatorial arguments exploit the fact that both Lewisians and Williamson subscribe to this necessitist principle. Seen from this perspective, the argument is a direct objection to first-order necessitism.

Is is tempting to conclude that the objection shows that first-order necessitists must reject the Urelement Set Axiom, and perhaps the iterative conception of set which some take to motivate the axiom. ${ }^{26}$ But in an expansionist setting, necessitists need not reject the set-theoretic principle. As one would anticipate, the expansionist rejects Moderate Plenitude but endorses a nearby, qualified principle:

Moderate Plenitude* For each cardinal number $v$, according to some at least as inclusive interpretation it is metaphysically possible that there exist $v$ non-sets.

This replacement principle speaks to whatever theoretical considerations initially led one to endorse Moderate Plenitude, and is compatible with the Urelement Set Axiom on both Lewis' concrete modal realism and Williamson's first-order necessitism. The moral is that when coupled with expansionism, the first-order necessitist is not committed to rejecting any principles of set theory by the recombinatorial argument.

To close, this final discussion suggests a broader project of integrating modal expansionism with a view on which 'set' is itself indefinitely extensible. Such an integrated view may have the potential to handle the modal set-theoretic recombinatorial

\footnotetext{
${ }^{25}$ See, for instance, Williamson [27, p. 17] and Divers [2] on this point.

${ }^{26}$ See Sider [21, p. 256] in particular for this latter point.
} 
paradoxes discussed in Hawthorne and Uzquiano [10], although this investigation must be left for future work.

Acknowledgments Thanks to Ben Brast-Mckie, Adrian Moore, and Ted Sider for discussions about earlier versions of this paper, and to Peter Fritz and an anonymous referee for this journal for their helpful and perceptive comments. Special thanks to James Studd and Timothy Williamson for their encouragement and comments on several drafts of this paper.

Open Access This article is distributed under the terms of the Creative Commons Attribution 4.0 International License (http://creativecommons.org/licenses/by/4.0/), which permits unrestricted use, distribution, and reproduction in any medium, provided you give appropriate credit to the original author(s) and the source, provide a link to the Creative Commons license, and indicate if changes were made.

\section{References}

1. Correia, F. (2007). Modality, quantification, and many Vlach-operators. Journal of Philosophical Logic, 36, 473-488.

2. Divers, J. (2014). Modal reality and (modal) logical space. Philosophy and Phenomenological Research, 88, 726-733.

3. Fine, K. (1977). Prior on the construction of possible worlds and instants, reprinted in K. Fine (Ed.) (2005) Modality and tense. Oxford: Clarendon Press.

4. Fine, K. (2002). Problem of Possibilia, reprinted in K. Fine (Ed.) (2005) Modality and tense. Oxford: Clarendon Press.

5. Fine, K. (2006). Relatively unrestricted quantification. In A. Rayo, \& G. Uzquiano (Eds.), Absolute generality. Oxford: Clarendon Press.

6. Forbes, G. (1992). Melia on modalism. Philosophical Studies, 68, 57-63.

7. Forster, T.E. (1995). Set theory with a universal set: exploring an untyped universe, 2nd edn. Oxford: Clarendon Press.

8. Fritz, P. (2016). A purely recombinatorial puzzle, forthcoming in Noûs.

9. Hawthorne, J. (2006). Quantity in Lewisian metaphysics. In J. Hawthorne (Ed.), Metaphysical essays. Oxford: Clarendon Press.

10. Hawthorne, J., \& Uzquiano, G. (2011). How many angels can dance on the point of a needle? Transcendental theology meets modal metaphysics. Mind, 123, 317-337.

11. Kaplan, D. (1995). A problem in possible-world semantics. In W. Sinnot-Armstrong (Ed.), Modality, morality, and belief: essays in honour of Ruth Barcan Marcus. Cambridge: Cambridge University Press.

12. Kratzer, A. (2012). Modals and conditionals. Oxford: Oxford University Press.

13. Lewis, D. (1986). On the plurality of worlds. Oxford: Basil Blackwell.

14. Linnebo, Ø. (2010). Pluralities and sets. Journal of Philosophy, 107, 144-164.

15. Melia, J. (1992). Against modalism. Philosophical Studies, 68, 35-56.

16. Nolan, D. (1996). Recombination unbound. Philosophical Studies, 84, 239-262.

17. Rayo, A. \& (MS) On the Open-Endedness of Logical Space, unpublished manuscript.

18. Rayo, A., \& Uzquiano, G. (Eds.) (2006). Absolute generality. Oxford: Clarendon Press.

19. Russell, B. (1906). On some difficulties in the theory of transfinite numbers and order types. Proceedings of The London Mathematics Society, 4, 29-53.

20. Salmon, N. (1989). The logic of what might have been. The Philosophical Review, 98, 3-34.

21. Sider, T. (2009). Williamson's many necessary existents. Analysis, 69, 50-58.

22. Studd, J. (2013). The iterative conception of set: a (bi-)modal axiomatisation. The Journal of Philosophical Logic, 42, 697-725.

23. Studd, J. (forthcoming). Everything, more or less: a defence of generality relativism, forthcoming with Oxford University Press.

24. Uzquiano, G. (2003). Plural quantification and classes. Philosophia Mathematica, 11, 67-81.

25. Uzquiano, G. (2015). Varieties of indefinite extensibility. Notre Dame Journal of Formal Logic, 56, 147-166. 
26. Warren, J. (2017). Quantifier variance and indefinite extensibility. Philosophical Review, 126, 81-122. 27. Williamson, T. (2013). Modal logic as metaphysics. Oxford: Oxford University Press.

28. Williamson, T. (2016). Modal science. Canadian Journal of Philosophy, 46, 453-492.

29. Yablo, S. (2006). Circularity and paradox. In T. Bolander, V.F. Hendricks, S.A. Pedersen (Eds.), Selfreference. CSLI Publications.

30. Yu, A. (2017). A modal account of propositions. Dialectica, 71, 463-488.

Publisher's Note Springer Nature remains neutral with regard to jurisdictional claims in published maps and institutional affiliations. 